VOL. 66 (2002) [267-273]

\title{
AN ESTIMATE FOR THE TOTAL MEAN CURVATURE IN NEGATIVELY CURVED SPACES
}

\author{
Albert BorbÉLY
}

Let $M^{n}$ be a nonpositively curved complete simply connected manifold and $D \subset$ $M^{n}$ be a convex compact subset with non-empty interior and smooth boundary. It is shown that the total mean curvature of $\partial D$ can be estimated in terms of volume and curvature bound.

\section{INTRODUCTION}

Mean curvature estimates and the isoperimetric conjecture for nonpositively curved manifolds are linked together by Kleiner's paper [3]. It was shown that the conjecture would follow from the following estimate.

$$
\int_{\partial D} H^{n-1} \geqslant \operatorname{Vol}\left(S^{n-1}\right)
$$

where $M^{n}$ is a nonpositively curved complete simply connected manifold, $D \subset M^{n}$ is a convex compact subset with non-empty interior and smooth boundary, $H$ denotes the mean curvature of $\partial D$ (the arithmetic mean of the principal curvatures) and $\operatorname{Vol}\left(S^{n-1}\right)$ is the Euclidean volume of the unit sphere.

This estimate is a simple consequence of the Gauss-Bonnet theorem in dimension three. The argument in [3] was carried out only in this case but it is not hard to see that it would work in higher dimensions as well. This gives a powerful motivation to prove $\left(^{*}\right)$ in higher dimensions. Unfortunately, in dimensions greater than three the Gauss-Bonnet-Chern integral does not seem to help. So one must find an alternative way.

The goal of the paper is to prove an estimate for the total mean curvature. Although it is not as strong as to imply $\left(^{*}\right)$ but it may be interesting on its own right.

THEOREM. Let $M^{n}$ be a nonpositively curved complete simply connected manifold with $n \geqslant 3$ and $D \subset M^{n}$ be a convex compact subset with non-empty interior and smooth boundary. Let $p \in D$ be an arbitrary point in the interior of $D$ and denote by

Received 21st February, 2002

Copyright Clearance Centre, Inc. Serial-fee code: 0004-9727/02 \$A2.00+0.00. 
$T$ the gradient vector field of the distance function from $p$. Then for the total mean curvature we have

$$
(n-1) \int_{\partial D} H \geqslant \int_{D}-\operatorname{Ric}(T)+2 \int_{D} \sum_{i<j} \lambda_{i} \lambda_{j}
$$

where $\operatorname{Ric}(T)$ is the Ricci tensor and $\lambda_{i}$ denotes the $i$ th principal curvature of the geodesic spheres around $p$.

The estimate is sharp when $D$ is a geodesic ball and $p \in D$ is the centre. Otherwise the right hand side will depend on the choice of $p \in D$. If we have a negative upper bound on the curvature, then the above estimate can be simplified as follows.

COROLLARY. Let $M^{n}$ be a negatively curved complete simply connected manifold with sectional curvatures $<-k^{2}$ and $n \geqslant 3$. Let $D \subset M^{n}$ be a convex compact subset with non-empty interior and smooth boundary. Then for the total mean curvature we have

$$
\int_{\partial D} H \geqslant(n-1) k^{2} \operatorname{Vol}(D)
$$

An alternative approach to prove $\left({ }^{*}\right)$ would be to estimate the total curvature of the boundary (the integral of the product of the mean curvatures) and use the inequality between the arithmetic and geometric means. Such estimate is obtained for the Hyperbolic space $H^{n}$ in [1].

\section{Construction of a differential Form}

We start out with a general construction that is similar to that of Chern's ([2]).

Let $e_{n}$ be a unit normal field defined on some open subset of $M^{n}$. At each point extend this to an orthonormal frame $e_{1}, \ldots, e_{n}$ such that $e_{i}$ is a smooth vector field for $i=1, \ldots, n$. This is possible locally although we may not be able to extend the frame smoothly over the whole of the open set. Denote by $\theta^{i}$ the dual frame of 1-forms and define the connection forms as

$$
\omega_{j}^{i}(X)=\left\langle\nabla_{X} e_{j}, e_{i}\right\rangle,
$$

where $\langle.,$.$\rangle denotes the metric on M^{n}$ and $X$ is a vector field. The curvature form is defined as

$$
\Omega_{j}^{i}(X, Y)=-\left\langle R(X, Y) e_{j}, e_{i}\right\rangle
$$

where $R(X, Y)$ denotes the curvature tensor defined as: $R(X, Y) Z=\nabla_{Y} \nabla_{X} Z$ $\nabla_{X} \nabla_{Y} Z+\nabla_{[X, Y]} Z$.

Then Cartan's equations read as

$$
\Omega_{j}^{i}=d \omega_{j}^{i}+\omega_{k}^{i} \omega_{j}^{k}, \quad d \theta^{i}=-\omega_{j}^{i} \theta^{j},
$$


where we use the usual summation convention, summing over repeated indices.

If we change the frame $e_{1}, \ldots, e_{n}$ into the frame $\widetilde{e}_{1}, \ldots, \widetilde{e}_{n-1}, \widetilde{e}_{n}=e_{n}$, then the corresponding forms will transform according to the following rules.

$$
\widetilde{e}_{i}=a_{j}^{i} e_{j}, \quad \widetilde{\theta}^{i}=a_{j}^{i} \theta^{j}, \quad \widetilde{\omega}_{n}^{i}=a_{j}^{i} \omega_{n}^{j}, \quad \widetilde{\Omega}_{n}^{i}=a_{j}^{i} \Omega_{n}^{j},
$$

where $i, j=1, \ldots, n-1$ and $a_{j}^{i}$ is an orthogonal matrix of smooth functions on $M^{n}$.

The differential form which is of interest to us is defined as:

$$
\Phi=\sum \varepsilon_{i_{1} \ldots i_{n-1}} \omega_{n}^{i_{1}} \theta^{i_{2}} \cdot \ldots \cdot \theta^{i_{n-1}},
$$

where $\varepsilon_{i_{1} \ldots i_{n-1}}$ is the Kronecker index that is equal to +1 or -1 according to whether the permutation $i_{1} \ldots i_{n-1}$ of the numbers $1,2, \ldots, n-1$ is even or odd and the summation is extended over all the indices $i_{1}, \ldots, i_{n-1}$ subject to the condition $i_{2}<i_{3}<\cdots<i_{n-1}$.

The forms $\theta^{i}, \omega_{n}^{i}$ and $\Omega_{n}^{i}$ are frame dependent but from the transformation formulas (2) one can easily see that the $(n-1)$-form $\Phi$ itself is independent of the choice of the local frame. This also means that $\Phi$ is defined globally (on the same open set where the vector field $e_{n}$ is defined) and depends only on the vector field $e_{n}$.

We defined $\Phi$ as an $(n-1)$-form on $M^{n}$ depending on a vector field $e_{n}$. But we shall need to look at $\Phi$ as a form in the unit tangent bundle $S M^{n}$. The forms $\theta^{i}, \omega_{j}^{i}$ and $\Omega_{j}^{i}$ can be regarded as forms in the unit tangent bundle $S M^{n}$. They will, of course, depend on how the frames $e_{1}, \ldots, e_{n-1}$ were chosen. To be more precise, if $e_{n} \in S M^{n}$ and $X, Y \in T_{e_{n}} S M^{n}$ are tangent vectors of $S M^{n}$ at $e_{n}$, then

$$
\Omega_{j}^{i}(X, Y)=-\left\langle R\left(X^{h}, Y^{h}\right) e_{j}, e_{i}\right\rangle,
$$

where $X^{h}, Y^{h}$ denotes the horizontal parts. To interpret the connection form $\omega_{j}^{i}(X)$ in case $X \in T_{e_{n}} S M^{n}$ we think of $X$ as a map $X:[0, \varepsilon) \rightarrow S M^{n}$ with $X(0)=e_{n}$. Then for any $t \in[0, \varepsilon)$ there is a frame $e_{1}, \ldots, e_{n}$ attached to $X(t)=e_{n}$. This gives rise to a map $X_{j}:[0, \varepsilon) \rightarrow S M^{n}$, where $X_{j}(t)=e_{j}$. Then we set $\omega_{j}^{i}(X)=\left\langle d / d t X_{j}(0), e_{i}\right\rangle$. In particular

$$
\omega_{n}^{i}(X)=\left\langle X^{v}, e_{i}\right\rangle
$$

where $X^{v}$ denotes the vertical part of $X$. For the form $\theta^{i}$ we simply have

$$
\theta^{i}(X)=\left\langle X^{h}, e_{i}\right\rangle
$$

With this in mind $\Phi$ can be regarded as an $(n-1)$-form on $S M^{n}$ which is independent of the choice of the frame $e_{1}, \ldots, e_{n-1}$ that was used to define the forms $\theta^{i}, \omega_{j}^{i}$ 
and $\Omega_{j}^{i}$. The form $\Phi$ (viewed as a form on $M^{n}$ ) is the pull back of $\Phi$ (viewed as a form on $S M^{n}$ ) from $S M^{n}$ via the map $e_{n}: M^{n} \rightarrow S M^{n}$. Since from the context it will be clear how to regard $\Phi$ we shall use the same notation for both forms.

From the equations (1) one can derive that

(6) $d \Phi=\sum \varepsilon_{i_{1} \ldots i_{n-1}} \Omega_{n}^{i_{1}} \theta^{i_{2}} \ldots \cdot \theta^{i_{n-1}}+2(-1)^{n-1} \sum \varepsilon_{i_{1} \ldots i_{n-1}} \omega_{n}^{i_{1}} \omega_{n}^{i_{2}} \theta^{i_{3}} \ldots \cdot \theta^{i_{n-1}} \theta^{n}$,

where the first summation is extended over all the indices $i_{1}, \ldots, i_{n-1}$ subject to the condition $i_{2}<i_{3}<\cdots<i_{n-1}$ and the second summation is extended over all the indices $i_{1}, \ldots, i_{n-1}$ subject to the conditions $i_{1}<i_{2}$ and $i_{3}<\cdots<i_{n-1}$.

\section{Proof OF ThEOREM 1.}

Let $p \in D$ be an arbitrary point in the interior of $D$. Denote by $T$ the gradient vector field of the distance function from $p$. Then $T$ is a smooth unit vector field on $M^{n}-\{p\}$. We can think of $T$ as a map $T: M^{n}-\{p\} \rightarrow S M^{n}$. Denote by $T(D)$ the image of $D$ under the map $T$, where we allow multiple values at $p$. More precisely,

$$
T(D)=T(D-\{p\}) \cup S_{p} M^{n},
$$

where $S_{p} M^{n}$ denotes the set of unit tangent vectors at $p$. This is a smooth $n$ dimensional submanifold of $S M^{n}$ with boundary $S_{p} M^{n} \cup T(\partial D)$.

Let us denote by $N$ the outer unit normal field of $\partial D$. Again we can consider it as a map $N: \partial D \rightarrow S M^{n}$. For a point $q \in \partial D$ denote by $[T(q), N(q)]$ the geodesic segment in $S M^{n}$ connecting the unit vectors $T(q)$ and $N(q)$. This is nothing but the set traced out by the rotation carrying the vector $T(q)$ into the vector $N(q)$ in $S_{q} M^{n}$.

Denote by $L_{q}$ the unit speed parametrisation of $[T(q), N(q)]$ and by $l_{q}$ the length of this segment. Let $\xi\left(e_{n}\right) \in T_{e_{n}} S M^{n}$ be the direction vector of $L_{q}$ at those points of $e_{n} \in[T(q), N(q)]$ where $T(q) \neq N(q)$. Then we define a map $R: \partial D \times[0,1] \rightarrow S M^{n}$ by $R(q, t)=L_{q}\left(l_{q} t\right)$. This is a smooth map and its image is denoted by $E$, which is the union of all these segments, namely:

$$
E=\bigcup_{q \in \partial D}[T(q), N(q)]
$$

Although $E$ is not a smooth $n$-dimensional submanifold of $S M^{n}$, for the map $R$ may not be one-to-one, we can integrate $n$-forms over $E$. Therefore Stokes' theorem will apply to $E$ with boundary $T(\partial D) \cup N(\partial D)$. We orient $T(D) \cup E$ in such a way that the orientation on $T(D)$ and $E$ will induce the opposite orientation on the common boundary $T(\partial D)$. Then by Stokes' theorem we have

$$
\int_{T(D) \cup E} d \Phi=\int_{S_{p} M^{n} \cup N(\partial D)} \Phi,
$$


where the boundary $S_{p} M^{n} \cup N(\partial D)$ is given the induced orientation.

Next, we are going to evaluate these integrals. Fix an orientation on $M^{n}$. The map $T: M^{n}-\{p\} \rightarrow S M^{n}$ induces an orientation on $T(D)$. Since $\Phi$ and therefore $d \Phi$ are independent of the choice of $e_{1}, \ldots, e_{n-1}$ to simplify the computations we select a special frame at each point.

Let $q \in D-\{p\}$ be an arbitrary point. Choose a positively oriented orthonormal frame $e_{1}, \ldots, e_{n}$ near $q$ such that $e_{n}=T$ and at the point $q$ the vectors $e_{1}, \ldots, e_{n-1}$ are principal directions for the geodesic sphere through $q$ centred around $p$. For the other points of the sphere the vectors $e_{1}, \ldots, e_{n-1}$ may no longer be principal directions. From the definition of the $\omega_{n}^{i}$ 's we have

$$
\omega_{n}^{i}\left(e_{n}\right)=0, \quad \omega_{n}^{i}\left(e_{j}\right)=\delta_{j}^{i} \lambda_{j}, \quad \text { for } \quad 1 \leqslant i, j \leqslant n-1
$$

at $q \in D-\{p\}$, where $\lambda_{j}$ denotes the principal curvature at $q$ of the geodesic sphere around $p$ in the direction of $e_{j}$ and $\delta_{j}^{i}$ is the Kronecker symbol.

We can now compute.

$$
\begin{aligned}
\int_{T(D)} d \Phi= & \int_{D} \sum \varepsilon_{i_{1} \ldots i_{n-1}} \Omega_{n}^{i_{1}} \theta^{i_{2}} \cdot \ldots \cdot \theta^{i_{n-1}}\left(e_{1}, \ldots, e_{n}\right) \\
& \quad+2(-1)^{n-1} \int_{D} \sum \varepsilon_{i_{1} \ldots i_{n-1}} \omega_{n}^{i_{1}} \omega_{n}^{i_{2}} \theta^{i_{3}} \ldots . \theta^{i_{n-1}} \theta^{n}\left(e_{1}, \ldots, e_{n}\right) \\
= & \int_{D} \sum \varepsilon_{i_{1} \ldots i_{n-1}} \varepsilon_{i_{1}, n, i_{2}, \ldots, i_{n-1}} K_{i_{1}, n}+2(-1)^{n-1} \int_{D} \sum \lambda_{i_{1}} \lambda_{i_{2}} \\
= & (-1)^{n-1}\left(\int_{D} \sum_{i_{1}=1}^{n-1}-K_{i_{1}, n}+2 \sum_{i_{1}<i_{2}} \lambda_{i_{1}} \lambda_{i_{2}}\right),
\end{aligned}
$$

where $K_{i_{1}, n}$ denotes the sectional curvature of the two-plane determined by $e_{i_{1}}, e_{n}$ and $i_{1}, i_{2}=1, \ldots, n-1$.

Next, we integrate over the set $E$. Let $e_{n} \in E \subset S M^{n}$ be an arbitrary unit vector. Then $e_{n} \in[T(q), N(q)]$ for some $q \in \partial D$. We choose the orientation for $\partial D \times[0,1]$ determined by the frame $f_{1}, \ldots, f_{n-1}, \partial / \partial t$, where the frame is chosen such that $f_{1}, \ldots, f_{n-1}$ is tangent to $\partial D$ and $f_{1}, \ldots, f_{n-1}, T$ is positively oriented in $M^{n}$. Then $E$ is given the orientation induced by the map $R$. It is easy to check that the orientations given to $E$ and $T(D)$ will induce the opposite orientation on the common boundary $T(\partial D)$.

To simplify the computation we choose the frame $f_{1}, \ldots, f_{n-1}$ in a special way. Denote by $A_{N}$ and $A_{T}$ the 2nd fundamental forms of $\partial D$ and the geodesic sphere around $p$ at the point $q$, respectively. These are positive definite endomorphisms of $N^{\perp}$ and $T^{\perp}$. Since $e_{n} \in[T(q), N(q)]$ we can write $e_{n}=a T+b N$ for some $a, b \geqslant 0$. 
Let $f_{2}, \ldots, f_{n-1}$ be a frame that diagonalises $a A_{N}+b A_{T}$ on the intersection $N^{\perp} \cap T^{\perp}$. This will be true only at the point $q \in \partial D$ and at nearby points $f_{2}, \ldots, f_{n-1}$ may not diagonalise $a A_{N}+b A_{T}$. Let $f_{1}$ be a unit vector tangent to $\partial D$ which is orthogonal to $f_{2}, \ldots, f_{n-1}$ and $\left\langle f_{1}, T\right\rangle \leqslant 0$. Moreover, we assume that the frame $f_{2}, \ldots, f_{n-1}$ was chosen in such a way that $f_{1}, \ldots, f_{n-1}, T$ is positively oriented in $M^{n}$. We now have

$$
\begin{aligned}
& \int_{E} d \Phi= \int_{\partial D \times[0,1]} d \Phi\left(d R\left(f_{1}\right), \ldots, d R\left(f_{n-1}\right), d R(\partial / \partial t)\right) \\
&= \int_{\partial D \times[0,1]} \sum \varepsilon_{i_{1} \ldots i_{n-1}} \Omega_{n}^{i_{1}} \theta^{i_{2}} \cdot \ldots \theta^{i_{n-1}}\left(d R\left(f_{1}\right), \ldots, d R\left(f_{n-1}\right), l_{q} \xi\right) \\
&+2(-1)^{n-1} \int_{\partial D \times[0,1]} \sum \varepsilon_{i_{1} \ldots i_{n-1}} \omega_{n}^{i_{1}} \omega_{n}^{i_{2}} \theta^{i_{3}} \cdot \ldots \cdot \theta^{i_{n-1}} \theta^{n} \\
&\left(d R\left(f_{1}\right), \ldots, d R\left(f_{n-1}\right), l_{q} \xi\right)
\end{aligned}
$$

where $d R(\partial / \partial t)=l_{q} \xi$.

Recall again that $d \Phi$ is independent of the choice $e_{1}, \ldots, e_{n-1}$. Therefore to evaluate the integrand we choose the frame $e_{1}, \ldots, e_{n}$ in such a way that $e_{i}=f_{i}$ for $i=2, \ldots, n-1$ and $\left\langle e_{1}, T\right\rangle \leqslant 0$. Since $e_{2}, \ldots, e_{n-1}$ diagonalise $a A_{N}+b A_{T}$ we have

$$
\omega_{n}^{i}\left(d R\left(f_{j}\right)\right)=\delta_{j}^{i} \beta_{j}, \quad \text { for } \quad 2 \leqslant i, j \leqslant n-1
$$

where $\beta_{j} \geqslant 0$ for $j=2, \ldots, n-1$ on account of the convexity of $D$. The tangent vector $\xi\left(e_{n}\right) \in T_{e_{n}} S M^{n}$ represents an infinitesimal rotation of $e_{n}$ in the direction of $e_{1}$ therefore it is a vertical vector and we also have

$$
\omega_{n}^{i}(\xi)=\delta_{1}^{i} .
$$

For the horizontal part of $\left(d R\left(f_{i}\right)\right)$ we have $\left(d R\left(f_{i}\right)\right)^{h}=f_{i}$ for $i=1, \ldots, n-1$ that implies $\theta^{i}\left(d R\left(f_{j}\right)\right)=\delta_{j}^{i}$ for $i, j=2, \ldots, n-1$.

Since $\Omega_{n}^{i_{1}} \theta^{i_{2}} \cdot \ldots \cdot \theta^{i_{n-1}}$ is a horizontal form and the tangent vector $\xi$ is vertical the integral in the second line of (10) is zero. Combining these observations with (11) and (12) we get

$$
\int_{E} d \Phi=2(-1)^{n-1} \int_{\partial D \times[0,1]} \sum_{i_{1}<i_{2}} \varepsilon_{i_{1}, \ldots, i_{n-1}} \varepsilon_{n, i_{2}, i_{3}, \ldots i_{n-1}, 1} \delta_{1}^{i_{1}} l_{q} \beta_{i_{2}} \theta^{n}\left(f_{1}\right) .
$$

From the choice of $f_{1}$ and $e_{1}$ one concludes that $f_{1}=c e_{1}-d e_{n}$ with $d \geqslant 0$, where $c$ and $d$ are functions of $e_{n} \in E$. Therefore $\theta^{n}\left(f_{1}\right)=-d$ and we have

$$
\int_{E} d \Phi=2(-1)^{n-1} \int_{\partial D \times[0,1]} \sum_{i_{2}=2}^{n-1} l_{q} \beta_{i_{2}} d .
$$


Next, we compute the integrals on the boundary $\partial(T(D) \cup E)=S_{p} M^{n} \cup N(\partial D)$. For the first component we obviously have

$$
\int_{S_{p} M^{n}} \Phi=0
$$

For the integration over $N(\partial D)$ first we fix a point $q \in \partial D$, then choose a positively oriented (in $M^{n}$ ) frame $e_{1}, \ldots, e_{n-1}, e_{n}$ at $q$ such that $e_{1}, \ldots, e_{n-1}$ are the principal directions for $\partial D$ at $q$. Then we have $\Phi\left(e_{1}, \ldots, e_{n-1}\right)=\sum_{1}^{n-1} \gamma_{i}$, where $\gamma_{i}$ denotes the principal curvature of $\partial D$ in the direction of $e_{i}$ at $q \in \partial D$. Since the induced orientation of $e_{1}, \ldots, e_{n-1}$ on $N(\partial D)$ is $\varepsilon_{n, 1, \ldots, n-1}=(-1)^{n-1}$ we have

$$
\int_{N(\partial D)} \Phi=(-1)^{n-1} \int_{\partial D} \sum_{1}^{n-1} \gamma_{i}
$$

Combining (7),(9),(13), (14) and (15) we get

$$
\int_{\partial D} \sum_{i=1}^{n-1} \gamma_{i}=\int_{D} \sum_{i_{1}=1}^{n-1}-K_{i_{1}, n}+\sum_{i_{1}<i_{2}} \lambda_{i_{1}} \lambda_{i_{2}}+\int_{\partial D \times[0,1]} \sum_{i_{2}=2}^{n-1} d l_{q} \beta_{i_{2}} .
$$

The last integral is non-negative since $d, \beta_{i} \geqslant 0$. This completes the proof of the Theorem.

If the sectional curvatures are bounded above by $-k^{2}$, we have $-K_{i, n}>k^{2}$ and $\lambda_{i}>k$. Putting these into (16) will yield the proof of the Corollary.

\section{REFERENCES}

[1] A. Borbély, 'On the total curvature of convex hypersurfaces in hyperbolic spaces', Proc. Amer. Math. Soc. (to appear).

[2] S-S. Chern, 'On the curvatura integra in a Riemannian manifold', Ann. of Math. 46 (1945), 674-684.

[3] B. Kleiner, 'An isoperimetric comparison theorem', Invent. Math. 108 (1992), 37-47.

Kuwait University

Department of Mathematics and Computer Science

P.O. Box 5969

Safat 13060

Kuwait e-mail: borbely@ mcs.sci.kuniv.edu.kw 